hep-th/0303239

IPM/P-2003/014

\title{
Off-shell extension of S-matrix elements and tachyonic effective actions
}

\author{
Mohammad R. Garousi \\ Department of Physics, Ferdowsi university, Mashhad, Iran \\ and \\ Institute for Studies in Theoretical Physics and Mathematics IPM \\ P.O. Box 19395-5531, Tehran, Iran
}

\begin{abstract}
We show that the on-shell S-matrix elements of four open string massless scalars, two scalars and two tachyons, and four open string tachyons in the super string theory can be written in a unique form. We then propose an off-shell extension for the S-matrix element of four scalars which is consistent, in the low energy limit, with the Dirac-Born-Infeld effective action. Using a similar off-shell extension for the S-matrix element of two scalars and two tachyons and for the S-matrix element of four tachyons, we show that they are fully consistent with the tachyonic DBI action.
\end{abstract}




\section{The idea}

Recently different tachyonic effective actions has been used to describe the time evolution of unstable D-branes in string theory $[1,2,3,4,5]^{1}$. In particular, Sen has shown that the string theory produces a pressure-less gas with non-zero energy density at the late time of the tachyon condensation[1]. In this paper, he showed that these results can be derived also from the tachyonic Dirac-Born-Infeld(DBI) effective action[7, 8] around its true vacuum. Other possible applications of this action to cosmology have been discussed in [9]. The tachyonic DBI action was first found by analyzing the low energy behaviour of the amplitude describing scattering of two tachyons to one graviton on the noncommutative world-volume of a non-BPS $\mathrm{D}_{p}$-branes in superstring theory [7]. Then it was shown that the action is consistent with T-duality rules, and has expected solitonic solutions[8].

In inferring effective action from on-shell string theory S-matrix elements, one usually evaluates the S-matrix elements and expands them in the limit $\alpha^{\prime} \rightarrow 0$, i.e., low energy limit. Then one writes a low energy effective action in the field theory that its corresponding on-shell S-matrix elements reproduce the leading order terms of the expansion [10]. The low energy expansion of the on-shell S-matrix elements are unique, however, many apparently different but physically identical low energy actions may produce them. These actions may in turn be related to each other by some field redefinition [11]. Alternatively, one may try to extend appropriately the on-shell S-matrix elements to different off-shell amplitudes and then expand them in the low energy limit. Each amplitude can be reproduced in field theory by a unique action. These actions should then be related to each other by some field redefinition. For example, consider the S-matrix element of one closed string tachyon and two open string tachyons in Type 0 theory $[12,7]$ :

$$
A(\tau, T, T) \sim\left(\frac{\Gamma(-2 s)}{\Gamma(-s) \Gamma(-s)}\right)
$$

where $\tau$ and $T$ stand for the closed string and open string tachyon, respectively. Also $s=-\alpha^{\prime}\left(k_{1}+k_{2}\right)^{2} / 2$, and $k_{1}^{a}, k_{2}^{a}$ are momenta of the open string tachyons which satisfy the on-shell condition $k_{1}^{2}=k^{2}=1 /\left(2 \alpha^{\prime}\right)$. One may analytically off-shell extends this amplitude to

$$
A^{\mathrm{off}}(\tau, T, T) \sim\left(\frac{\Gamma(-2 s)}{\Gamma(-s) \Gamma(-s)}+1 / 4-\alpha^{\prime}\left(k_{1}^{2}+k_{2}^{2}\right) / 4+f\left(k_{1}, k_{2}\right)\right)
$$

where the tachyon momenta do not satisfy the on-shell condition anymore, and the function $f\left(k_{1}, k_{2}\right)$, having only some contact terms of order $O\left(\alpha^{\prime 2}\right)$, must be symmetric under $1 \leftrightarrow 2$ and vanishes on-shell. Obviously the off-shell amplitude (2) reduces to the on-shell amplitude (1) upon imposing the on-shell condition $k_{1}^{2}=k_{2}^{2}=1 /\left(2 \alpha^{\prime}\right)$. The low energy

\footnotetext{
${ }^{1}$ For early studies of open string tachyon dynamics, see [6].
} 
expansion, i.e., $\alpha^{\prime} \rightarrow 0$, of $(2)$ is

$$
A^{\text {off }}(\tau, T, T) \sim\left(1 / 4+\alpha^{\prime} k_{1} \cdot k_{2} / 2+O\left(\alpha^{2}\right)\right) .
$$

The first two terms are reproduced exactly by the tachyonic DBI action[7, 8]2

$$
\begin{aligned}
S & =-\frac{T_{p}}{4} \int d x \tau V(T) \sqrt{-\operatorname{det}\left(\eta_{a b}+2 \pi \alpha^{\prime} \partial_{a} T \partial_{b} T\right)}, \\
& =-\frac{T_{p}}{4} \int d x \tau\left(1-\pi T^{2} / 2+\pi \alpha^{\prime}(\partial T)^{2}+\cdots\right),
\end{aligned}
$$

where in the second line we have used the expansion $V(T)=1-\frac{\pi}{2} T^{2}+O\left(T^{4}\right)$ at the top of the tachyon potential in this action. Note that to evaluate the off-shell S-matrix elements in field theory we do not use the on-shell conditions for external states in Feynman diagrams, however, we do use the conservation of momentum. The terms of order $O\left(\alpha^{\prime 2}\right)$ in $(3)$ are related to higher derivative terms which are not included in tachyonic action above ${ }^{3}$. Now consider the following off-shell extension of the amplitude (1):

$$
A^{\text {off }}(\tau, T, T) \sim \frac{\Gamma\left(1+2 \alpha^{\prime} k_{1} \cdot k_{2}\right)}{\Gamma\left(1 / 2+\alpha^{\prime} k_{1} \cdot k_{2}\right) \Gamma\left(1 / 2+\alpha^{\prime} k_{1} \cdot k_{2}\right)}+g\left(k_{1}, k_{2}\right)
$$

where function $g\left(k_{1}, k_{2}\right)$, having only some contact terms of order $O\left(\alpha^{\prime 2}\right)$, is zero on-shell. Again it is obvious that this off-shell amplitude reduces to the on-shell amplitude (1) upon imposing the on-shell condition. At low energy this amplitude has the following expansion:

$$
A^{\text {off }} \sim 1 / 4+\alpha^{\prime} \ln (2) k_{1} \cdot k_{2}+O\left(\alpha^{\prime 2}\right)
$$

In this case, the first two terms of this expansion are reproduced exactly by BSFT action[14]

$$
\begin{aligned}
S & =-\frac{T_{p}}{4} \int d x \tau e^{-T^{2} / 4} \frac{\sqrt{\pi} \Gamma\left(\alpha^{\prime}(\partial T)^{2} / 2+1\right)}{\Gamma\left(\alpha^{\prime}(\partial T)^{2} / 2+1 / 2\right)} \\
& =-\frac{T_{p}}{4} \int d x \tau\left(1-T^{2} / 4+\alpha^{\prime} \ln (2)(\partial T)^{2}+\cdots\right)
\end{aligned}
$$

Again the terms of order $O\left(\alpha^{\prime 2}\right)$ in (5) are related to the higher derivative terms which are not included in the BSFT action. One expects that the two actions (4) and (6) to be related to each other by some field redefinition[15].

Now using the idea that one can off-shell extend the S-matrix elements, the question is how to off-shell extend a S-matrix element? The particular case that we are interested in is how to off-shell extend S-matrix elements involving open string tachyons? The guiding principle that we follow is the similarity between on-shell S-matrix elements involving

\footnotetext{
${ }^{2}$ We have used the fact that the closed string tachyon in Type 0 theory normalizes the D-brane tension as $T_{p} \rightarrow T_{p}(1+\tau / 4+\cdots)[16]$, and we have kept only linear term for the closed string tachyon.

${ }^{3}$ The higher derivative terms may have significant effect at the top of potential, however, according to the results in [1] they are not important at the minimum of tachyon potential.
} 
massless scalars and the on-shell S-matrix elements involving tachyons. We extend this similarity to the off-shell amplitudes as well. We off-shell extend the amplitudes involving only scalars in such a way that they are consistent with the DBI action in the low energy limit. Then we use a similar off-shell amplitude for the cases that involve tachyons. As a simple example consider the S-matrix element of one closed string tachyon and two open string massless scalar states. The amplitude is given by $[13]^{4}$,

$$
A(\tau, X, X) \sim \zeta_{1} \cdot \zeta_{2}\left(\frac{\Gamma(-2 s)}{\Gamma(-s) \Gamma(-s)}\right)
$$

where $s=-\alpha^{\prime}\left(k_{1}+k_{2}\right)^{2} / 2$, and $\zeta_{1}^{i}, \zeta_{2}^{i}$ and $k_{1}^{a}, k_{2}^{a}$ are the scalar polarizations and momenta, respectively ${ }^{5}$. The momenta in this amplitude satisfy the on-shell condition $k_{1}^{2}=k_{2}^{2}=0$. Note that apart from the polarization of the scalar fields, the on-shell amplitude (1) and (7) are similar. Off-shell extension for the above amplitude which is correspond to the DBI action is

$$
A^{\text {off }}(\tau, X, X) \sim \zeta_{1} \cdot \zeta_{2}\left(\frac{\Gamma(-2 s)}{\Gamma(-s) \Gamma(-s)}+G-G^{\text {on }}+h\left(k_{1}, k_{2}\right)\right)
$$

where the function $G\left(k_{1}, k_{2}\right)$ is

$$
G=-\alpha^{\prime}\left(k_{1}^{2}+k_{2}^{2}\right) / 4
$$

and $G^{\text {on }}$ means the function $G$ in which the momenta are on-shell. Obviously it is zero in this case. The momenta in (8) do not satisfy the on-shell conditions and the function $h\left(k_{1}, k_{2}\right)$, having only some contact terms of order $O\left(\alpha^{\prime 2}\right)$, must be symmetric under $1 \leftrightarrow 2$ and must be zero on-shell. This function is related to the higher derivative terms in the DBI action. The low energy expansion, i.e., $\alpha^{\prime} \rightarrow 0$, of this amplitude is

$$
A^{\text {off }} \sim \zeta_{1} \cdot \zeta_{2}\left(\alpha^{\prime} k_{1} \cdot k_{2} / 2+O\left(\alpha^{\prime 2}\right)\right)
$$

The first term above is reproduced in field theory by the DBI action as required, and $O\left(\alpha^{2}\right)$ terms are related to the higher derivative terms which are not included in the DBI action. Now the off-shell amplitude (2) is similar to the off-shell amplitude (8), i.e.,

$$
A^{\text {off }}(\tau, T, T) \sim\left(\frac{\Gamma(-2 s)}{\Gamma(-s) \Gamma(-s)}+G-G^{\text {on }}+f\left(k_{1}, k_{2}\right)\right) .
$$

In this case $G^{\text {on }}=-1 / 4$. This off-shell amplitude is consistent with the tachyonic DBI action as we saw above. In the present paper we would like to extend these idea to the

\footnotetext{
${ }^{4}$ Using the fact that the closed strings are not functional of the open string tachyon but are functional of the scalar fields [22], we assumed that the closed string field does not depend on the scalar. This makes similar the S-matrix elements involving the scalars and the tachyon as much as possible.

${ }^{5}$ Our index conventions are that early Latin indices take values in the world-volume, e.g., $a, b=0,1, \ldots, p$, and middle Latin indices take values in the transverse space, e.g., $i, j=p+1, \ldots, 8,9$.
} 
case of S-matrix elements of four tachyons and/or scalars in the superstring theory in the presence of background B-flux.

In section 2, we recall the string theory S-matrix element of four scalar vertex operators. We propose an off-shell extension for this amplitude and then expand it at low energy. We show that its leading order terms are reproduced exactly by the corresponding off-shell amplitude in field theory based on the DBI action. In section 3, we show that the on-shell S-matrix element of two scalar and two tachyon vertex operators is similar to the on-shell S-matrix element of four scalars. Hence we write an off-shell S-matrix elements that is similar to the one in section 2. In section 4 we do the same thing for the S-matrix element of four tachyons. The proposed off-shell amplitudes in sections 3 and 4 are correspond to the tachyonic DBI action.

\section{Four scalars amplitude}

Scattering amplitude of four vector vertex operators in superstring theory is evaluated in [18], and its low energy effective action is also studied, for example, in [19]. To find the amplitude corresponding to four scalar vertex operators, one may use the result in [18] in which the vector polarizations $\zeta^{a}$ are replaced by the scalar polarizations $\zeta^{i}$. Since we are interested in the scattering amplitude in the presence of B-flux, one should use $G=\left(1 /\left(\eta+2 \pi \alpha^{\prime} B\right)\right)_{S}$ as the world volume metric, and also should add an appropriate phase factor to the amplitudes in one cycling of the vertex operators with the non-commutative parameter tensor $\theta=\left(2 \pi \alpha^{\prime} /\left(\eta+2 \pi \alpha^{\prime} B\right)\right)_{A}[20]$. Adding all non-cyclic permutation of the vertex operators, one ends up with the following amplitude:

$$
A\left(\zeta_{1}, \zeta_{2}, \zeta_{3}, \zeta_{4}\right)=A_{s}\left(\zeta_{1}, \zeta_{2}, \zeta_{3}, \zeta_{4}\right)+A_{u}\left(\zeta_{1}, \zeta_{2}, \zeta_{3}, \zeta_{4}\right)+A_{t}\left(\zeta_{1}, \zeta_{2}, \zeta_{3}, \zeta_{4}\right),
$$

where $A_{s}, A_{u}$, and $A_{t}$ are the part of the amplitude that has massless pole in $s^{-}, u$ - and $t$-channels, respectively. They are

$$
\begin{aligned}
A_{s}= & -\frac{i c T_{p}}{2 \pi^{2} \alpha^{\prime 2}} \zeta_{1} \cdot \zeta_{2} \zeta_{3} \cdot \zeta_{4}\left(\frac{\Gamma(-2 s) \Gamma(1-2 t)}{\Gamma(-2 s-2 t)}\left(e^{i \pi l_{12}+i \pi l_{34}}+e^{i \pi l_{14}-i \pi l_{23}}\right)\right. \\
& +\frac{\Gamma(-2 s) \Gamma(1-2 u)}{\Gamma(-2 s-2 u)}\left(e^{i \pi l_{13}-i \pi l_{24}}+e^{i \pi l_{12}-i \pi l_{34}}\right) \\
& \left.-\frac{\Gamma(1-2 t) \Gamma(1-2 u)}{\Gamma(1-2 t-2 u)}\left(e^{i \pi l_{14}+i \pi l_{23}}+e^{i \pi l_{13}+i \pi l_{24}}\right)\right), \\
A_{u}= & -\frac{i c T_{p}}{2 \pi^{2} \alpha^{\prime 2} \zeta_{1} \cdot \zeta_{3} \zeta_{2} \cdot \zeta_{4}}\left(-\frac{\Gamma(1-2 s) \Gamma(1-2 t)}{\Gamma(1-2 s-2 t)}\left(e^{i \pi l_{12}+i \pi l_{34}}+e^{i \pi l_{14}-i \pi l_{23}}\right)\right. \\
& +\frac{\Gamma(-2 u) \Gamma(1-2 s)}{\Gamma(-2 u-2 s)}\left(e^{i \pi l_{13}-i \pi l_{24}}+e^{i \pi l_{12}-i \pi l_{34}}\right)
\end{aligned}
$$




$$
\begin{aligned}
& \left.+\frac{\Gamma(-2 u) \Gamma(1-2 t)}{\Gamma(-2 u-2 t)}\left(e^{i \pi l_{14}+i \pi l_{23}}+e^{i \pi l_{13}+i \pi l_{24}}\right)\right) \\
A_{t}= & -\frac{i c T_{p}}{2 \pi^{2} \alpha^{\prime 2}} \zeta_{1} \cdot \zeta_{4} \zeta_{2} \cdot \zeta_{3}\left(\frac{\Gamma(-2 t) \Gamma(1-2 s)}{\Gamma(-2 t-2 s)}\left(e^{i \pi l_{12}+i \pi l_{34}}+e^{i \pi l_{14}-i \pi l_{23}}\right)\right. \\
& -\frac{\Gamma(1-2 s) \Gamma(1-2 u)}{\Gamma(1-2 s-2 u)}\left(e^{i \pi l_{13}-i \pi l_{24}}+e^{i \pi l_{12}-i \pi l_{34}}\right) \\
& \left.+\frac{\Gamma(-2 t) \Gamma(1-2 u)}{\Gamma(-2 t-2 u)}\left(e^{i \pi l_{14}+i \pi l_{23}}+e^{i \pi l_{13}+i \pi l_{24}}\right)\right)
\end{aligned}
$$

where $l_{\alpha \beta}=k_{\alpha} \cdot \theta \cdot k_{\beta} /(2 \pi)$ for $\alpha, \beta=1,2,3,4$, and variables $s, t, u$ are the following:

$$
\begin{aligned}
s & =-\alpha^{\prime}\left(k_{1}+k_{2}\right)^{2} / 2, \\
t & =-\alpha^{\prime}\left(k_{2}+k_{3}\right)^{2} / 2, \\
u & =-\alpha^{\prime}\left(k_{1}+k_{3}\right)^{2} / 2 .
\end{aligned}
$$

Using on-shell condition for the momenta, one finds that these Mandelstam variables satisfy the on-shell relation $s+t+u=0$. We have also normalized the amplitude by the factor $i c T_{p} /\left(2 \pi^{2} \alpha^{2}\right)$ where $c=\sqrt{-\operatorname{det}(\eta+B)}$. The amplitudes $A_{s}, A_{u}$ and $A_{t}$ are very similar, so we only off-shell extend the $A_{t}$ amplitude. The off-shell extension of $A_{s}$ and $A_{u}$ should then be straightforward.

To find the off-shell extension of $A_{t}$ we use the following criteria: 1)-The amplitude should have poles at $2 t=0,1,2, \cdots$, corresponding to propagation of the infinite tower of open string states in the amplitude.

2)-Imposing on-shell conditions, the amplitude must reduce to the on-shell amplitude $A_{t}$. 3)-In the low energy expansion of the amplitude, its massless pole and its contact terms up to $O\left(\alpha^{\prime 3}\right)$ should produce the corresponding pole and contact terms of the DBI field theory amplitude.

Unlike the amplitude (7) in which the Mandelstam variable $s$ is arbitrary, the Mandelstam variables in (10) are constraint by the on-shell condition $s+t+u=0$. Now it raises the question that how we write the gamma functions in (10) in the off-shell amplitude. To do so, we first, using the on-shell condition $s+t+u=0$, write the on-shell amplitude in a unique form that all other amplitudes involving the tachyon can also be converted to. It turns out that the gamma function in $A_{t}$ should be written in the following form:

$$
\begin{aligned}
\frac{\Gamma(-2 t) \Gamma(1-2 s)}{\Gamma(-2 t-2 s)} & =\frac{\Gamma(-2 t) \Gamma(1+t+u-s)}{\Gamma(u-s-t)} \\
\frac{\Gamma(1-2 s) \Gamma(1-2 u)}{\Gamma(1-2 s-2 u)} & =\frac{\Gamma(1+t+u-s) \Gamma(1+s+t-u)}{\Gamma(1+2 t)} \\
\frac{\Gamma(-2 t) \Gamma(1-2 u)}{\Gamma(-2 t-2 u)} & =\frac{\Gamma(-2 t) \Gamma(1+t+s-u)}{\Gamma(s-u-t)}
\end{aligned}
$$


Writing the gamma functions in (10) in the above form, we propose the following off-shell amplitude for $A_{t}$ :

$$
\begin{aligned}
A_{t}^{\text {off }}= & -\frac{i c T_{p}}{2 \pi^{2} \alpha^{\prime 2}} \zeta_{1} \cdot \zeta_{4} \zeta_{2} \cdot \zeta_{3} \\
& \times\left(\left(\frac{\Gamma(-2 t) \Gamma(1+t+u-s)}{\Gamma(u-s-t)}+F-F^{\mathrm{on}}+f\right)\left(e^{i \pi l_{12}+i \pi l_{34}}+e^{i \pi l_{14}-i \pi l_{23}}\right)\right. \\
& -\left(\frac{\Gamma(1+t+u-s) \Gamma(1+s+t-u)}{\Gamma(1+2 t)}-F+F^{\mathrm{on}}-g\right)\left(e^{i \pi l_{13}-i \pi l_{24}}+e^{i \pi l_{12}-i \pi l_{34}}\right) \\
& \left.+\left(\frac{\Gamma(-2 t) \Gamma(1+t+s-u)}{\Gamma(s-u-t)}+F-F^{\mathrm{on}}+h\right)\left(e^{i \pi l_{14}+i \pi l_{23}}+e^{i \pi l_{13}+i \pi l_{24}}\right)\right)
\end{aligned}
$$

where the function $F\left(k_{1}, k_{2}, k_{3}, k_{4}\right)$ has been added to the amplitude to cancel the non-desire contact terms resulting from expansion of the gamma functions in the low energy limit. It has the following contact terms of order $\alpha^{\prime 2}$ :

$$
\begin{aligned}
F= & -\frac{\alpha^{\prime} \pi^{2}}{6}\left(\alpha^{\prime}\left(\sum_{i=1}^{4} k_{i}^{2}\right)^{2} / 4+\left(k_{1}^{2}+k_{4}^{2}\right) t+\left(k_{1}^{2}+k_{3}^{2}\right) u+\left(k_{1}^{2}+k_{2}^{2}\right) s\right. \\
& \left.+\alpha^{\prime} k_{2} \cdot k_{3}\left(k_{1}^{2}+k_{4}^{2}\right)-\alpha^{\prime} k_{1} \cdot k_{3}\left(k_{2}^{2}+k_{4}^{2}\right)-\alpha^{\prime} k_{1} \cdot k_{2}\left(k_{3}^{2}+k_{4}^{2}\right)\right) .
\end{aligned}
$$

$F^{\text {on }}$ means function $F$ in which the momenta are on-shell. It is easy to see that this is zero in this case. Functions $f\left(k_{1}, k_{2}, k_{3}, k_{4}\right), g\left(k_{1}, k_{2}, k_{3}, k_{4}\right), h\left(k_{1}, k_{2}, k_{3}, k_{4}\right)$, having only some contact terms of order $O\left(\alpha^{\prime 3}\right)$, must be zero on-shell. These functions are related to the higher derivative corrections to the DBI action in which we are not interested in the present paper. Using conservation of momentum, the Mandelstam variables in the off-shell case satisfy the relation:

$$
s+t+u=-\alpha^{\prime}\left(\sum_{i=1}^{4} k_{i}^{2}\right) / 2 .
$$

Its is obvious that this off-shell amplitude satisfies the first two criteria above. To check the last criterion, we should expand the amplitude in the low energy. The gamma functions in this amplitude at $\alpha^{\prime} \rightarrow 0$ have the following expansion:

$$
\begin{aligned}
\frac{\Gamma(-2 t) \Gamma(1+t+u-s)}{\Gamma(u-s-t)} & =\frac{1}{2}+\frac{s-u}{2 t}-\frac{\pi^{2}}{6}\left((s-u)^{2}-t^{2}\right)+O\left(\alpha^{\prime 3}\right), \\
\frac{\Gamma(-2 t) \Gamma(1+t+s-u)}{\Gamma(s-u-t)} & =\frac{1}{2}+\frac{u-s}{2 t}-\frac{\pi^{2}}{6}\left((s-u)^{2}-t^{2}\right)+O\left(\alpha^{\prime 3}\right), \\
\frac{\Gamma(1+t+u-s) \Gamma(1+s+t-u)}{\Gamma(1+2 t)} & =1+\frac{\pi^{2}}{6}\left((s-u)^{2}-t^{2}\right)+O\left(\alpha^{\prime 3}\right) .
\end{aligned}
$$

Replacing these expansion for the gamma functions into (13), one finds massless pole and some contact terms at each order of $\alpha^{\prime}$. 
Now in field theory, using the non-commutative DBI action, one finds the following massless $t$-channel amplitude (see e.g., [13] for details):

$$
\begin{aligned}
A_{t}^{\prime \text { off }}= & \left(\widetilde{V}_{\phi_{2} \phi_{3} A}\right)^{a}\left(\widetilde{G}_{A}\right)_{a b}\left(\widetilde{V}_{A \phi_{1} \phi_{4}}\right)^{b}, \\
= & \left(\frac{i c T_{p}}{\left(\alpha^{\prime} \pi\right)^{2}}\right) \zeta_{1} \cdot \zeta_{4} \zeta_{2} \cdot \zeta_{3} \frac{\sin \left(\pi l_{23}\right) \sin \left(\pi l_{14}\right) \alpha^{\prime}\left(k_{2}-k_{3}\right) \cdot\left(k_{1}-k_{4}\right)}{2 t}, \\
= & \frac{-i c T_{p}}{2 \pi^{2} \alpha^{\prime 2}} \zeta_{1} \cdot \zeta_{4} \zeta_{2} \cdot \zeta_{3}\left(\left(e^{i \pi l_{12}+i \pi l_{34}}+e^{i \pi l_{14}-i \pi l_{23}}\right)\left(\frac{s-u}{2 t}\right)\right. \\
& \left.\quad+\left(e^{i \pi l_{14}+i \pi l_{23}}+e^{i \pi l_{13}+i \pi l_{24}}\right)\left(\frac{u-s}{2 t}\right)\right) .
\end{aligned}
$$

In reaching to this result, we have used only conservation of momentum. It easy to see that the massless pole of the string theory off-shell amplitude (13) produces exactly the corresponding amplitude in the field theory. Now subtracting the field theory massless pole from the string theory amplitude, one ends up with the following contact terms in the $t$-channel:

$$
\begin{aligned}
A_{t}^{\text {off }}-A_{t}^{\text {off }}= & -\frac{i c T_{p}}{2 \pi^{2} \alpha^{\prime 2}} \zeta_{1} \cdot \zeta_{4} \zeta_{2} \cdot \zeta_{3} \\
& \times\left(\left(e^{i \pi l_{12}+i \pi l_{34}}+e^{i \pi l_{14}-i \pi l_{23}}\right)\left(\frac{1}{2}-\frac{\pi^{2}}{6}\left((s-u)^{2}-t^{2}\right)+F+O\left(\alpha^{\prime 3}\right)\right)\right. \\
& +\left(e^{i \pi l_{13}-i \pi l_{24}}+e^{i \pi l_{12}-i \pi l_{34}}\right)\left(-1-\frac{\pi^{2}}{6}\left((s-u)^{2}-t^{2}\right)+F+O\left(\alpha^{\prime 3}\right)\right) \\
& \left.+\left(e^{i \pi l_{14}+i \pi l_{23}}+e^{i \pi l_{13}+i \pi l_{24}}\right)\left(\frac{1}{2}-\frac{\pi^{2}}{6}\left((s-u)^{2}-t^{2}\right)+F+O\left(\alpha^{\prime 3}\right)\right)\right) .
\end{aligned}
$$

Doing the same analysis for the $A_{s}$ and $A_{u}$, one finds the following total off-shell contact terms:

$$
A_{c}^{\text {off }}\left(\zeta_{1}, \zeta_{2}, \zeta_{3}, \zeta_{4}\right)=A_{c}^{0}\left(\zeta_{1}, \zeta_{2}, \zeta_{3}, \zeta_{4}\right)+A_{c}^{4}\left(\zeta_{1}, \zeta_{2}, \zeta_{3}, \zeta_{4}\right)+\sum_{n>4} A_{c}^{n}\left(\zeta_{1}, \zeta_{2}, \zeta_{3}, \zeta_{4}\right),
$$

where $A_{c}^{0}$ contains, apart from the phase factor, contact terms with no momentum and is zero when the background B-flux vanishes,

$$
\begin{aligned}
A_{c}^{0}= & \frac{-i c T_{p}}{2 \pi^{2} \alpha^{\prime 2}}\left(e^{i \pi l_{12}+i \pi l_{34}}+e^{i \pi l_{14}-i \pi l_{23}}\right)\left(\frac{1}{2} \zeta_{1} \cdot \zeta_{4} \zeta_{2} \cdot \zeta_{3}+\frac{1}{2} \zeta_{1} \cdot \zeta_{2} \zeta_{3} \cdot \zeta_{4}-\zeta_{1} \cdot \zeta_{3} \zeta_{2} \cdot \zeta_{4}\right) \\
& -\frac{i c T_{p}}{2 \pi^{2} \alpha^{\prime 2}}\left(e^{i \pi l_{13}-i \pi l_{24}}+e^{i \pi l_{12}-i \pi l_{34}}\right)\left(-\zeta_{1} \cdot \zeta_{4} \zeta_{2} \cdot \zeta_{3}+\frac{1}{2} \zeta_{1} \cdot \zeta_{2} \zeta_{3} \cdot \zeta_{4}+\frac{1}{2} \zeta_{1} \cdot \zeta_{3} \zeta_{2} \cdot \zeta_{4}\right) \\
& -\frac{i c T_{p}}{2 \pi^{2} \alpha^{\prime 2}}\left(e^{i \pi l_{14}+i \pi l_{23}}+e^{i \pi l_{13}+i \pi l_{24}}\right)\left(\frac{1}{2} \zeta_{1} \cdot \zeta_{4} \zeta_{2} \cdot \zeta_{3}-\zeta_{1} \cdot \zeta_{2} \zeta_{3} \cdot \zeta_{4}+\frac{1}{2} \zeta_{1} \cdot \zeta_{3} \zeta_{2} \cdot \zeta_{4}\right) \cdot(19)
\end{aligned}
$$

$A_{c}^{4}$ contains terms with four momenta which are non vanishing even in the $B=0$ limit. They are

$$
A_{c}^{4}=\frac{-i c T_{p}}{6}\left(e^{i \pi l_{12}+i \pi l_{34}}+e^{i \pi l_{14}-i \pi l_{23}}+e^{i \pi l_{13}-i \pi l_{24}}+e^{i \pi l_{12}-i \pi l_{34}}+e^{i \pi l_{14}+i \pi l_{23}}+e^{i \pi l_{13}+i \pi l_{24}}\right)
$$




$$
\begin{aligned}
& \times\left(\zeta_{1} \cdot \zeta_{4} \zeta_{2} \cdot \zeta_{3}\left(\left(k_{2} \cdot k_{3}\right)\left(k_{1} \cdot k_{4}\right)-\left(k_{1} \cdot k_{2}\right)\left(k_{3} \cdot k_{4}\right)-\left(k_{1} \cdot k_{3}\right)\left(k_{2} \cdot k_{4}\right)\right)\right. \\
& +\zeta_{1} \cdot \zeta_{2} \zeta_{3} \cdot \zeta_{4}\left(\left(k_{1} \cdot k_{2}\right)\left(k_{3} \cdot k_{4}\right)-\left(k_{2} \cdot k_{3}\right)\left(k_{1} \cdot k_{4}\right)-\left(k_{1} \cdot k_{3}\right)\left(k_{2} \cdot k_{4}\right)\right) \\
& \left.+\zeta_{1} \cdot \zeta_{3} \zeta_{2} \cdot \zeta_{4}\left(\left(k_{1} \cdot k_{3}\right)\left(k_{2} \cdot k_{4}\right)-\left(k_{1} \cdot k_{2}\right)\left(k_{3} \cdot k_{4}\right)-\left(k_{2} \cdot k_{3}\right)\left(k_{1} \cdot k_{2}\right)\right)\right) .
\end{aligned}
$$

And $A_{c}^{n}$ with $n>4$ contains contact terms with more than four momenta. We refer readers to [21] for comparing the contact terms $A_{c}^{0}$ and $A_{c}^{4}$ with the noncommutative DBI action. Here we consider only the simple case that the background field $B=0$. In this case, $A_{c}^{0}=0$, the phase factors in $A_{c}^{4}$ reduce to number 6 and $c \rightarrow 1$. Expanding the square root of determinant, one finds that the tachyonic DBI action has the following four scalars coupling:

$$
\begin{aligned}
S & =-T_{p} \int d^{p+1} x V(T) \sqrt{-\operatorname{det}\left(\eta_{a b}+\partial_{a} X^{i} \partial_{b} X_{i}+2 \pi \alpha^{\prime} \partial_{a} T \partial_{b} T\right)} \\
& =-T_{p} \int d^{p+1} x\left(-\frac{1}{4}\left(\partial_{a} X^{i} \partial_{b} X_{i}\right)\left(\partial^{b} X_{j} \partial^{a} X^{j}\right)+\frac{1}{8}\left(\partial_{a} X^{i} \partial^{a} X_{i}\right)^{2}+\cdots\right) .
\end{aligned}
$$

It is easy to verify that couplings above exactly reproduce the contact terms of $A_{c}^{4}$ when $B=0$. Note that in reaching to this result, one does not need to use the on-shell conditions for external states. This confirms that the off-shell amplitude (13) is consistent with the DBI action.

\section{Two scalars and two tachyons amplitude}

The amplitude describing scattering of two tachyons to two scalars on the world-volume of a non-commutative non-BPS $\mathrm{D}_{p}$-brane is evaluated in [21]. This amplitude has only massless pole in the $t$-channel, that is

$$
\begin{aligned}
A\left(\zeta_{2}, \zeta_{3}\right)=A_{t}\left(\zeta_{2}, \zeta_{3}\right)= & -\frac{i c T_{p}}{\pi \alpha^{\prime}} \zeta_{2} \cdot \zeta_{3}\left(\frac{\Gamma(-2 t) \Gamma(1 / 2-2 s)}{\Gamma(-1 / 2-2 t-2 s)}\left(e^{i \pi l_{12}+i \pi l_{34}}+e^{i \pi l_{14}-i \pi l_{23}}\right)\right. \\
& -\frac{\Gamma(1 / 2-2 s) \Gamma(1 / 2-2 u)}{\Gamma(-2 s-2 u)}\left(e^{i \pi l_{13}-i \pi l_{24}}+e^{i \pi l_{12}-i \pi l_{34}}\right) \\
& \left.+\frac{\Gamma(-2 t) \Gamma(1 / 2-2 u)}{\Gamma(-1 / 2-2 t-2 u)}\left(e^{i \pi l_{14}+i \pi l_{23}}+e^{i \pi l_{13}+i \pi l_{24}}\right)\right)
\end{aligned}
$$

where we have normalized the amplitude here by the factor $i c T_{p} /\left(\pi \alpha^{\prime}\right)$. The Mandelstam variables $s, t, u$ defined in (11), and they satisfy the on-shell condition $s+t+u=-1 / 2$.

Using relation $s+t+u=-1 / 2$ in (22), one can write the gamma functions in (22) as those appearing in (12), i.e.,

$$
\frac{\Gamma(-2 t) \Gamma(1 / 2-2 s)}{\Gamma(-1 / 2-2 t-2 s)}=\frac{\Gamma(-2 t) \Gamma(1+t+u-s)}{\Gamma(u-s-t)},
$$




$$
\begin{aligned}
\frac{\Gamma(1 / 2-2 s) \Gamma(1 / 2-2 u)}{\Gamma(-2 s-2 u)} & =\frac{\Gamma(1+t+u-s) \Gamma(1+s+t-u)}{\Gamma(1+2 t)} \\
\frac{\Gamma(-2 t) \Gamma(1 / 2-2 u)}{\Gamma(-1 / 2-2 t-2 u)} & =\frac{\Gamma(-2 t) \Gamma(1+t+s-u)}{\Gamma(s-u-t)} .
\end{aligned}
$$

Using these form for the gamma functions in (22), one can see that the on-shell amplitudes (22) and (10) can be written in exactly the same form. Now using the guiding principle the the off-shell amplitude should also be similar, we propose the following off-shell extension for the amplitude (22):

$$
\begin{aligned}
A_{t}^{\mathrm{off}}= & -\frac{i c T_{p}}{\pi \alpha^{\prime}} \zeta_{2} \cdot \zeta_{3}\left(\left(\frac{\Gamma(-2 t) \Gamma(1+t+u-s)}{\Gamma(u-s-t)}+F-F^{\mathrm{on}}+f^{\prime}\right)\left(e^{i \pi l_{12}+i \pi l_{34}}+e^{i \pi l_{14}-i \pi l_{23}}\right)\right. \\
& -\left(\frac{\Gamma(1+t+u-s) \Gamma(1+s+t-u)}{\Gamma(1+2 t)}-F+F^{\mathrm{on}}-g^{\prime}\right)\left(e^{i \pi l_{13}-i \pi l_{24}}+e^{i \pi l_{12}-i \pi l_{34}}\right) \\
& \left.+\left(\frac{\Gamma(-2 t) \Gamma(1+t+s-u)}{\Gamma(s-u-t)}+F-F^{\mathrm{on}}+h^{\prime}\right)\left(e^{i \pi l_{14}+i \pi l_{23}}+e^{i \pi l_{13}+i \pi l_{24}}\right)\right),
\end{aligned}
$$

where $F$ is given by (14) in which $k_{2}, k_{3}$ are momenta of the scalar states and $k_{1}, k_{4}$ are momenta of the tachyons. $F^{\text {on }}$ means function $F$ in which the momenta are on-shell. It has the following value:

$$
F^{\text {on }}=-\frac{\pi^{2}}{6} \alpha^{\prime} k_{2} \cdot k_{3}
$$

Functions $f^{\prime}\left(k_{1}, k_{2}, k_{3}, k_{4}\right), g^{\prime}\left(k_{1}, k_{2}, k_{3}, k_{4}\right), h^{\prime}\left(k_{1}, k_{2}, k_{3}, k_{4}\right)$ in (24) must be zero on-shell. We expect them to be related to the higher derivative terms in the tachyonic DBI action in which we are not interested in this paper. So from now on we ignore them. The Madelstam variables in the off-shell amplitude (24) satisfy (15). Obviously the amplitude (24) has expected infinite tower of poles in the $t$-channel, and reduces to (22) upon imposing the on-shell conditions.

Using the low energy expansion (16) for the gamma functions in (24) one finds massless pole and some contact terms at each order of $\alpha^{\prime}$. Now in field theory, since the kinetic term of tachyon in the tachyonic DBI action (21) is exactly like the kinetic term of the massless scalar field, with different normalization i.e., $X^{i} \rightarrow \sqrt{2 \pi \alpha^{\prime}} T$, the massless $t$-channel pole for scattering two scalars to two tachyons is exactly like the off-shell amplitude (17) in which $\zeta_{1} \cdot \zeta_{4} \rightarrow 2 \pi \alpha^{\prime}$. This pole is exactly the massless pole of the string theory amplitude (24). Subtracting the massless pole of (24), one finds the following contact terms:

$$
A_{c}^{\text {off }}\left(\zeta_{2}, \zeta_{3}\right)=A_{c}^{0}\left(\zeta_{2}, \zeta_{3}\right)+A_{c}^{4}\left(\zeta_{2}, \zeta_{3}\right)+\cdots,
$$

where $A_{c}^{0}$ includes the contact terms that have no momentum and is zero when the background field vanishes,

$$
A_{c}^{0}=-\frac{i c T_{p}}{\pi \alpha^{\prime}} \zeta_{2} \cdot \zeta_{3}\left(\frac{1}{2}\left(e^{i \pi l_{12}+i \pi l_{34}}+e^{i \pi l_{14}-i \pi l_{23}}\right)\right.
$$




$$
\left.-\left(e^{i \pi l_{13}-i \pi l_{24}}+e^{i \pi l_{12}-i \pi l_{34}}\right)+\frac{1}{2}\left(e^{i \pi l_{14}+i \pi l_{23}}+e^{i \pi l_{13}+i \pi l_{24}}\right)\right) .
$$

$A_{c}^{4}$ includes contact terms that have at most four momenta and is non-zero even when $B=0$, i.e.,

$$
\begin{aligned}
A_{c}^{4}= & \frac{-4 i \pi c T_{p}}{3 \alpha^{\prime}}\left(e^{i \pi l_{12}+i \pi l_{34}}+e^{i \pi l_{14}-i \pi l_{23}}+e^{i \pi l_{13}-i \pi l_{24}}+e^{i \pi l_{12}-i \pi l_{34}}+e^{i \pi l_{14}+i \pi l_{23}}+e^{i \pi l_{13}+i \pi l_{24}}\right) \\
& \times \zeta_{2} \cdot \zeta_{3}\left(\frac{\alpha^{\prime 2}}{4}\left(k_{2} \cdot k_{3}\right)\left(k_{1} \cdot k_{4}\right)-\frac{\alpha^{\prime 2}}{4}\left(k_{1} \cdot k_{2}\right)\left(k_{3} \cdot k_{4}\right)-\frac{\alpha^{\prime 2}}{4}\left(k_{1} \cdot k_{3}\right)\left(k_{2} \cdot k_{4}\right)+\frac{\alpha^{\prime}}{8}\left(k_{2} \cdot k_{3}\right)\right)(28)
\end{aligned}
$$

The dots in $(26)$ are the $O\left(\alpha^{\prime 3}\right)$ terms of the gamma function expansion and the $f^{\prime}, g^{\prime}, h^{\prime}$ functions in (24). Again we refer readers to [21] for comparing the contact terms $A_{c}^{0}$ and $A_{c}^{4}$ above with the noncommutative DBI action. Here we consider only the simple case that the background field $B=0$. The tachyonic DBI action (21) has the following terms:

$$
\begin{aligned}
\mathcal{L}(X, X, T, T)= & -T_{p}\left(-\frac{\pi}{4} T^{2}\left(\partial_{a} X^{i} \partial^{a} X_{i}\right)\right. \\
& \left.-\pi \alpha^{\prime}\left(\partial_{a} X^{i} \partial_{b} X_{i}\right)\left(\partial^{b} T \partial^{a} T\right)+\frac{\pi \alpha^{\prime}}{2}\left(\partial_{a} X^{i} \partial_{a} X_{i}\right)\left(\partial_{b} T \partial^{b} T\right)\right),
\end{aligned}
$$

It is easy to see that the coupling in the first line produce the term in (28) which has two momenta, and the couplings in the second line above reproduce the other terms in (28). This confirms that the off-shell amplitude (24) is consistent with the tachyonic DBI action, and the expectation that the function $f^{\prime}, g^{\prime}, h^{\prime}$ are related to the higher derivative terms in this action, i.e., their $\alpha^{\prime}$ expansion does not have constant and term proportional to $\alpha^{\prime}$.

\section{Four tachyons amplitude}

The amplitude describing scattering of two tachyons to two other tachyons on the worldvolume of a non-commutative non-BPS $\mathrm{D}_{p}$-brane is evaluated in [21]. This amplitude has massless pole in all $s^{-}, t$ - and $u$-channels, that is,

$$
A=A_{s}+A_{u}+A_{t}
$$

However, in this case the amplitudes in all channels are identical i.e., $A_{s}=A_{t}=A_{u}$, and

$$
\begin{aligned}
A_{t}= & -2 i c T_{p}\left(\frac{\Gamma(-2 t) \Gamma(-2 s)}{\Gamma(-1-2 s-2 t)}\left(e^{i \pi l_{12}+i \pi l_{34}}+e^{i \pi l_{14}-i \pi l_{23}}\right)\right. \\
& -\frac{\Gamma(-2 s) \Gamma(-2 u)}{\Gamma(-1-2 s-2 u)}\left(e^{i \pi l_{13}-i \pi l_{24}}+e^{i \pi l_{12}-i \pi l_{34}}\right) \\
& \left.+\frac{\Gamma(-2 t) \Gamma(-2 u)}{\Gamma(-1-2 t-2 u)}\left(e^{i \pi l_{14}+i \pi l_{23}}+e^{i \pi l_{13}+i \pi l_{24}}\right)\right),
\end{aligned}
$$


where we have normalized the amplitude by the factor $2 i c T_{p}$. The variables $s, t, u$ satisfy the on-shell condition $s+t+u=-1$.

Again using relation $s+t+u=-1$ in (30), one can write the gamma functions in it as those appearing in (12), i.e.,

$$
\begin{aligned}
\frac{\Gamma(-2 t) \Gamma(-2 s)}{\Gamma(-1-2 t-2 s)} & =\frac{\Gamma(-2 t) \Gamma(1+t+u-s)}{\Gamma(u-s-t)} \\
\frac{\Gamma(-2 s) \Gamma(-2 u)}{\Gamma(-1-2 s-2 u)} & =\frac{\Gamma(1+t+u-s) \Gamma(1+s+t-u)}{\Gamma(1+2 t)} \\
\frac{\Gamma(-2 t) \Gamma(-2 u)}{\Gamma(-1-2 t-2 u)} & =\frac{\Gamma(-2 t) \Gamma(1+t+s-u)}{\Gamma(s-u-t)} .
\end{aligned}
$$

Using these form for the gamma functions in (30), (23) for the gamma functions in (22) and (12) for the gamma functions in (10), one can easily see that the on-shell amplitudes (30), (22) and (10) can be written in exactly the same form. Now using the guiding principle that the off-shell amplitudes should also be similar, we propose the following off-shell extension for the amplitude (30):

$$
\begin{aligned}
A_{t}^{\mathrm{off}}= & -2 i c T_{p}\left(\left(\frac{\Gamma(-2 t) \Gamma(1+t+u-s)}{\Gamma(u-s-t)}+F-F^{\mathrm{on}}+f^{\prime \prime}\right)\left(e^{i \pi l_{12}+i \pi l_{34}}+e^{i \pi l_{14}-i \pi l_{23}}\right)\right. \\
& -\left(\frac{\Gamma(1+t+u-s) \Gamma(1+s+t-u)}{\Gamma(1+2 t)}-F+F^{\mathrm{on}}-g^{\prime \prime}\right)\left(e^{i \pi l_{13}-i \pi l_{24}}+e^{i \pi l_{12}-i \pi l_{34}}\right) \\
& \left.+\left(\frac{\Gamma(-2 t) \Gamma(1+t+s-u)}{\Gamma(s-u-t)}+F-F^{\mathrm{on}}+h^{\prime \prime}\right)\left(e^{i \pi l_{14}+i \pi l_{23}}+e^{i \pi l_{13}+i \pi l_{24}}\right)\right), \quad(31)
\end{aligned}
$$

where $F$ is given by (14) in which all the momenta are the tachyon momenta. $F^{\text {on }}$ in this case is

$$
F^{\mathrm{on}}=-\frac{\pi^{2}}{6}\left(\alpha^{\prime} k_{2} \cdot k_{3}+\alpha^{\prime} k_{1} \cdot k_{4}+\frac{1}{2}\right) .
$$

Functions $f^{\prime \prime}\left(k_{1}, k_{2}, k_{3}, k_{4}\right), g^{\prime \prime}\left(k_{1}, k_{2}, k_{3}, k_{4}\right), h^{\prime \prime}\left(k_{1}, k_{2}, k_{3}, k_{4}\right)$ must be zero on-shell. We expect them to be related to the higher derivative terms in the tachyonic DBI action in which we are not interested in the present paper. So we ignore them from now on. It is obvious that the off-shell amplitude (31) has the expected infinite tower of poles in the $t$-channel, and reduces to (30) upon imposing on-shell conditions.

Using the low energy expansion (16) for the gamma functions in (31), one finds massless pole and some contact terms at each order of $\alpha^{\prime}$. In field theory, the massless pole is again exactly like (17) in which $\zeta_{2} \cdot \zeta_{3} \zeta_{1} \cdot \zeta_{4} \rightarrow\left(2 \pi \alpha^{\prime}\right)^{2}$. Subtracting it from the off-shell amplitude (31) and repeating the same analysis for $A_{s}$ and $A_{u}$, one finds the following total contact terms:

$$
A_{c}^{\text {off }}=-\frac{8 \pi^{2} i c T_{p}}{3}
$$




$$
\begin{aligned}
& \times\left(e^{i \pi l_{12}+i \pi l_{34}}+e^{i \pi l_{14}-i \pi l_{23}}+e^{i \pi l_{13}-i \pi l_{24}}+e^{i \pi l_{12}-i \pi l_{34}}+e^{i \pi l_{14}+i \pi l_{23}}+e^{i \pi l_{13}+i \pi l_{24}}\right) \\
& \times\left(-\frac{\alpha^{\prime 2}}{4}\left(k_{2} \cdot k_{3}\right)\left(k_{1} \cdot k_{4}\right)-\frac{\alpha^{\prime 2}}{4}\left(k_{1} \cdot k_{2}\right)\left(k_{3} \cdot k_{4}\right)-\frac{\alpha^{\prime 2}}{4}\left(k_{1} \cdot k_{3}\right)\left(k_{2} \cdot k_{4}\right)\right. \\
& \left.+\frac{\alpha^{\prime}}{8}\left(k_{2} \cdot k_{3}+k_{1} \cdot k_{4}+k_{1} \cdot k_{3}+k_{2} \cdot k_{4}+k_{1} \cdot k_{2}+k_{3} \cdot k_{4}\right)+\frac{3}{16}\right)+\cdots
\end{aligned}
$$

where dots represent the $O\left(\alpha^{\prime 3}\right)$ terms of the gamma function and functions $f^{\prime \prime}, g^{\prime \prime}, h^{\prime \prime}$. Here again to compare it with the tachyonic DBI action for the case that $B=0$, one should replace the phase factor above by number 6 and $c \rightarrow 1$. The action (21) has the following terms:

$$
\mathcal{L}(T, T, T, T)=-T_{p}\left(\beta T^{4}-\frac{\pi^{2} \alpha^{\prime}}{2} T^{2}\left(\partial_{a} T \partial^{a} T\right)-\frac{\pi^{2} \alpha^{\prime 2}}{2}\left(\partial_{a} T \partial^{a} T\right)^{2}\right)
$$

where the constant $\beta$ is the coefficient of $T^{4}$ in the tachyon potential. One can easily observe that the term with four derivatives reproduces exactly the four momentum contact terms in (33), term with two derivatives reproduces the two momentum contact terms, and the $T^{4}$ term produces the constant term in (33) provided that $\beta=\pi^{2} / 8$.

The tachyon potential expanded around its maximum, i.e., around $T_{\max }=0$, has then the following expansion:

$$
V(T)=1-\frac{\pi}{2} T^{2}+\frac{\pi^{2}}{8} T^{4}+O\left(T^{6}\right)
$$

On the other hand A. Sen has shown that the tachyon potential in the tachyonic DBI action has minimum at $T_{\min } \rightarrow \infty$, and the behavior of the potential around the minimum should be like $e^{-\sqrt{\pi} T}[23]^{6}$. A tachyon potential with this behavior at $T_{\min } \rightarrow \infty$, and expansion (35) at the maximum of potential has been suggested in [21]. Our calculation however does not rule out the possibility of other expansion for the potential. This stems from the fact that functions $f^{\prime \prime}, g^{\prime \prime}, h^{\prime \prime}$ in (31) which must be zero on-shell, might have a constant term in their $\alpha^{\prime}$ expansion. Another potential which has the above expansion around maximum is the following:

$$
V(T)=e^{-\pi T^{2} / 2}
$$

This potential is consistent with the sigma model effective action[17].

Extending the similarity between the tachyon and scalar to the higher derivative terms as well, one might expect that the functions $f^{\prime}, g^{\prime}, h^{\prime}$ in (24) to be related to the functions $f, g, h$ in (13) as $f^{\prime}=f-f^{\text {on }}, g^{\prime}=g-g^{\text {on }}, h^{\prime}=h-h^{\text {on }}$, and similarly the functions $f^{\prime \prime}, g^{\prime \prime}, h^{\prime \prime}$ in $(31)$ as $f^{\prime \prime}=f-f^{\text {on }}, g^{\prime \prime}=g^{\text {on }}, h^{\prime \prime}=h^{\text {on }}$. This means that the higher derivative

\footnotetext{
${ }^{6}$ Note that the convention in [23] sets $\alpha^{\prime}=1$.
} 
corrections to the tachyonic DBI action and the higher derivative terms of DBI action have the same structure. Similar speculation, in other context, has been also made in [24]

Finally to compare the low energy expansion of the off-shell amplitudes considered in this paper with the on-shell amplitudes considered in [21], one should not that in the offshell case the Mandelstam variables are all independent. Hence at low energy they all go to zero, as we have considered in this paper. Whereas the on-shell relation like (15) constrains the variables $s, t, u$ not to be independent anymore in the on-shell amplitudes. In this case one should write the amplitudes in terms of only independent variables then expands them at the low energy. For instance, as it has been done in [21], for studying $A_{t}$ at low energy one writes $t$ in terms of $s$ and $u$. Then expands the amplitude at $\alpha^{\prime} k_{i} \cdot k_{j} \rightarrow 0$. In this regards, both the off-shell amplitudes proposed in this paper and the on-shell amplitudes gives the same low energy expansion.

Acknowledgement: I would like to thank M. Alishahiha, A. Sen and A.A. Tseytlin for comments.

\section{References}

[1] A. Sen, "Tachyon Matter," arXiv:hep-th/0203265.

[2] G. W. Gibbons, Phys. Lett. B 537, 1(2002) [arXiv:hep-th/0204008].

[3] S. Sugimoto and S. Terashima, JHEP 0207, 025 (2002) [arXiv:hep-th/0205085].

[4] J. A. Minahan, JHEP 0207, 030 (2002) [arXiv:hep- th/0205098].

[5] N. D. Lambert and I. Sachs, "Tachyon Dynamics and the Effective Action Approximation", arXiv:hep-th/0208217.

[6] K. Bardakci, Nucl. Phys. B 68 (1974) 331; Nucl. Phys. B 133 (1978) 297;

K. Bardakci and M. B. Halpern, Phys. Rev. D 10 (1974) 4230; Nucl. Phys. B 96 (1975) 285.

[7] M. R. Garousi, Nucl. Phys. B 584, 284 (2000) [arXiv:hep-th/0003122].

[8] E. A. Bergshoeff, M. de Roo, T. C. de Wit, E. Eyras and S. Panda, JHEP 0005, 009 (2000) [arXiv:hep-th/0003221];

J. Kluson, Phys. Rev. D 62, 126003 (2000) [arXiv:hep-th/0004106].

[9] S. Alexander, "Inflation fron D-anti-D brane annihilation," arXiv:hep-th/0105032; A. Mazumdar, S. Panda and A. Perez-Lorenzana, "Assisted inflation via tachyon condensation," Nucl. Phys. B 614(2001) [arXiv:hep-th/0107058]; M. Fairbairn and 
M. H. Tytgat, "Inflation from a tachyon fluid?," Phys. Lett. B 546, 1 (2002) [arXiv:hepth/0204070]; S. Mukohyama, "Brane cosmology driven by the rolling tachyon," Phys. Rev. D 66, 024009 (2002) [arXiv:hep-th/0204084]; A. Feinstein, "Power-law inflation from the rolling tachyon," Phys. Rev. D 66, 063511 (2002) [arXiv:hep-th/0204140]; T. Padmanabhan, "Accelerated expansion of the universe driven by tachyonic matter," Phys. Rev. D 66, 021301 (2002) [arXiv:hep-th/0204150]; A. V. Frolov, L. Kofman and A. A. Starobinsky, "Prospects and problems of tachyon matter cosmology," Phys. Lett. B 545, 8 (2002) [arXiv:hep-th/0204187]; D. Choudhury, D. Ghoshal, D. P. Jatkar and S. Panda, "On the cosmological relevance of the tachyon," Phys. Lett. B 544, 231 (2002) [arXiv:hep-th/0204204]; X. z. Li, J. g. Hao and D. j. Liu, "Can quintessence be the rolling tachyon?," Chin. Phys. Lett. 19, 1584 (2002) [arXiv:hep-th/0204252]; G. Shiu and I. Wasserman, "Cosmological constraints on tachyon matter," Phys. Lett. B 541, 6 (2002) [arXiv:hep-th/0205003]; L. Kofman and A. Linde, "Problems with tachyon inflation," JHEP 0207, 004 (2002) [arXiv:hep-th/0205121]; H. B. Benaoum, "Accelerated universe from modified Chaplygin gas and tachyonic fluid," arXiv:hepth/0205140; M. Sami, "Implementing power law inflation with rolling tachyon on the brane," arXiv:hep-th/0205146; M. Sami, P. Chingangbam and T. Qureshi, "Aspects of tachyonic inflation with exponential potential," Phys. Rev. D 66, 043530 (2002) [arXiv:hep-th/0205179]; J. c. Hwang and H. Noh, "Cosmological perturbations in a generalized gravity including tachyonic condensation," Phys. Rev. D 66, 084009 (2002) [arXiv:hep-th/0206100]; T. Mehen and B. Wecht, "Gauge fields and scalars in rolling tachyon backgrounds," arXiv:hep-th/0206212; K. Ohta and T. Yokono, "Gravitational approach to tachyon matter," Phys. Rev. D 66, 125009 (2002) [arXiv:hep-th/0207004]; Y. S. Piao, R. G. Cai, X. m. Zhang and Y. Z. Zhang, "Assisted tachyonic inflation," Phys. Rev. D 66, 121301 (2002) [arXiv:hep-ph/0207143]; G. Shiu, S. H. Tye and I. Wasserman, "Rolling tachyon in brane world cosmology from superstring field theory," arXiv:hep-th/0207119; X. z. Li, D. j. Liu and J. g. Hao, "On the tachyon inflation," arXiv:hep-th/0207146; J. M. Cline, H. Firouzjahi and P. Martineau, "Reheating from tachyon condensation," JHEP 0211, 041 (2002) [arXiv:hep-th/0207156]; A. Buchel, P. Langfelder and L. Walcher, "Does the Tachyon Matter," Annals Phys. 302 (2002) 78 [arXiv:hep-th/0207235]; G. N. Felder, L. Kofman and A. Starobinsky, "Caustics in tachyon matter and other Born-Infeld scalars," JHEP 0209, 026 (2002) [arXiv:hep-th/0208019]; B. Wang, E. Abdalla and R. K. Su, "Dynamics and holographic discreteness of tachyonic inflation," arXiv:hep-th/0208023; S. Mukohyama, "Inhomogeneous tachyon decay, light-cone structure and D-brane network problem in tachyon cosmology," Phys. Rev. D 66, 123512 (2002) [arXiv:hep-th/0208094]; M. C. Bento, O. Bertolami and A. A. Sen, "Tachyonic inflation in the braneworld scenario," arXiv:hep-th/0208124; J. g. Hao and X. z. Li, "Reconstructing the equation of state of tachyon," Phys. Rev. D 66, 087301 (2002) [arXiv:hep-th/0209041]; C. j. Kim, H. B. Kim and Y. b. Kim, "Rolling tachyons in string cosmology," Phys. Lett. B 552, 111 (2003) [arXiv:hep-th/0210101]; F. Quevedo, "Lectures on string / brane cosmology," Class. Quant. Grav. 19, 5721 (2002) [arXiv:hep-th/0210292]; G. Shiu, "Tachyon 
dynamics and brane cosmology," arXiv:hep-th/0210313; J. S. Bagla, H. K. Jassal and T. Padmanabhan, "Cosmology with tachyon field as dark energy," arXiv:astroph/0212198; Y. S. Piao, Q. G. Huang, X. m. Zhang and Y. Z. Zhang, "Non-minimally coupled tachyon and inflation," arXiv:hep-ph/0212219; X. z. Li and X. h. Zhai, "The tachyon inflationary models with exact mode functions," arXiv:hep-ph/0301063; G. W. Gibbons, "Thoughts on tachyon cosmology," arXiv:hep-th/0301117; M. Sami, P. Chingangbam and T. Qureshi, "Cosmological aspects of rolling tachyon," arXiv:hepth/0301140; C. j. Kim, H. B. Kim, Y. b. Kim and O. K. Kwon, "Cosmology of rolling tachyon," arXiv:hep-th/0301142; F. Leblond and A. Peet, "SD-brane gravity fields and rolling tachyon," arXiv:hep-th/0303035.

[10] J. Scherk, Nucl. Phys. B 31 (1971) 222;

A. Neveu and J. Scherk, Nucl. Phys. B 36 (1972) 155;

J. Scherk and J.H. Schwarx, Nucl. Phys. B 81 (1974) 118;

T. Yoneya, Prog. Theor. Phys. 51 (1974) 1907.

[11] A. A. Tseytlin, Phys. Lett. B 176 (1986) 92;

R. R. Metsaev and A. A. Tseytlin, Phys. Lett. B 185 (1987) 52.

[12] A. Hashimoto and I. R. Klebanov, Phys. Lett. B 381 (1996) 437 [arXiv:hepth/9604065].

[13] M. R. Garousi, Nucl. Phys. B 579 (2000) 209 [arXiv:hep-th/9909214].

[14] D. Kutasov, M. Marino and G. W. Moore, JHEP 0010, 045 (2000) [arXiv:hepth/0009148];

D. Kutasov, M. Marino and G. W. Moore, arXiv:hep-th/0010108;

P. Kraus and F. Larsen, Phys. Rev. D 63, 106004 (2001) [arXiv:hep-th/0012198];

T. Takayanagi, S. Terashima and T. Uesugi, JHEP 0103, 019 (2001) [arXiv:hepth/0012210].

[15] P. Mukhopadhyay and A. Sen, arXiv:hep-th/0208142.

[16] I. Klebanov and A. A. Tseytlin, Nucl. Phys. B 547 (1999) 143 [arXiv:hep-th/9812089]; M. R. Garousi, Nucl. Phys. B 550 (1999) 225 [arXiv:hep-th/9901085].

[17] A. A. Tseytlin, J. Math. Phys. 42 (2001) 2854 [arXiv:hep-th/0011033].

[18] M. B. Green and J. H. Schwarz, Nucl. Phys. B 198 (1982)252; 198 (1982) 441;

I. H. Schwarz, Phys. Reports 89 (1982) 223.

[19] A. A. Tseytlin, Nucl. Phys. B 276 (1986) 391.

[20] N. Seiberg and E. Witten, JHEP 9909, 032 (1999) [arXiv:hep-th/9908142].

[21] M. R. Garousi, Nucl. Phys. B 647 (2002) 117 [arXiv:hep-th/0209068] 
[22] M. R. Garousi and R. C. Myers, Nucl. Phys. B 542 (1999) 73 [raXiv:hep-th/9809100].

[23] A. Sen, "Field Theory of Tachyon Matter," arXiv:hep-th/0204143.

[24] A. Sen, "Dirac-Born-Infeld Action on the Tachyon Kink and Vortex" arXiv:hepth/0303057. 